\title{
Reexamining the possible benefits of visual crowding: dissociating crowding from ensemble percepts
}

\author{
Paul F. Bulakowski • Robert B. Post • David Whitney
}

Published online: 9 February 2011

(C) The Author(s) 2011. This article is published with open access at Springerlink.com

\begin{abstract}
Peripheral objects and their features become indistinct when closely surrounding but nonoverlapping objects are present. Most models suggest that this phenomenon, called crowding, reflects limitations of visual processing, but an intriguing idea is that it may be, in part, adaptive. Specifically, the mechanism generating crowding may simultaneously facilitate ensemble representations of features, leaving meaningful information about clusters of objects. In two experiments, we tested whether visual crowding and the perception of ensemble features share a common mechanism. Observers judged the orientation of a crowded bar, or the ensemble orientation of all bars in the upper and lower visual fields. While crowding was predictably stronger in the upper relative to the lower visual field, the ensemble percept did not vary between the visual fields. Featural averaging within the crowded region does not always scale with the resolution limit defined by crowding, suggesting that dissociable processes contribute to visual crowding and ensemble percepts.
\end{abstract}

Keywords Crowding $\cdot$ Ensemble perception $\cdot$ Mean extraction $\cdot$ Visual resolution

\author{
P. F. Bulakowski $\cdot$ R. B. Post $\cdot$ D. Whitney \\ Department of Psychology, University of California, \\ Davis 95616, USA \\ P. F. Bulakowski $\cdot$ D. Whitney \\ Center for Mind and Brain, University of California, \\ Davis 95616, USA \\ P. F. Bulakowski $(\bowtie) \cdot$ D. Whitney \\ Vision Science Group, University of California, \\ Berkeley, USA \\ e-mail: pbulakowski@ucdavis.edu \\ D. Whitney \\ Department of Psychology, University of California, \\ Berkeley, California 97420, USA
}

Perceiving an object accurately in peripheral vision becomes exceedingly difficult when similar objects are nearby - a phenomenon known as visual crowding (Bouma, 1970; Stuart \& Burian, 1962; Townsend, Taylor, \& Brown, 1971). Flanking objects appear to "squash" the crowded target (Korte, 1923, as cited in Levi, 2008), and crowds of objects form an indistinct or unidentifiable jumble of features (Pelli, 2008). The degree of crowding increases with eccentricity (Bouma, 1970) and is more pronounced in the upper visual field (UVF), relative to the lower visual field (LVF; He, Cavanagh, \& Intriligator, 1996). Crowding may result from a number of mechanisms: contour integration (Flom, Weymouth, \& Kahneman, 1963); lateral interactions (Bouma, 1970); insufficiently small integration fields (Pelli, Palomares, \& Majaj, 2004); coarse (He et al., 1996), unfocused, or mislocalized attention (Strasburger, 2005); or averaging (pooling) of target-flanker featural information (Parkes, Lund, Angelucci, Solomon, \& Morgan, 2001). Crowding might also occur independently at different stages of visual analysis (Farzin, Rivera, \& Whitney, 2009; Louie, Bressler \& Whitney, 2007). Whether there is any beneficial by-product of crowding remains an open question.

When multiple objects fill a scene, observers can report the average, or global, property of the set with high precision (Bulakowski, Bressler, \& Whitney, 2007; Dakin \& Watt, 1997; Watamaniuk \& Sekuler, 1992; Williams \& Sekuler, 1984). The perception of average, or ensemble, characteristics is beneficial, since it provides information about the texture and the gist of a scene. Perceiving ensemble characteristics is possible when multiple objects are densely packed or are widely spaced and easily individuated (Ariely, 2001; Chong \& Treisman, 2003). Ensemble perception results from the extraction of statistical properties of low-level features (e.g., size, orientation, and motion; Ariely, 2001; Parkes et al., 2001; Watamaniuk 
\& Sekuler, 1992) or even high-level, holistically processed objects and attributes (e.g., faces and emotions; Haberman \& Whitney, 2007). It has been speculated that ensemble perception is beneficial by alleviating the need to process and represent individual items at a higher level (Ariely, 2001; Haberman, Harp, \& Whitney, 2009; Haberman \& Whitney, 2007, 2009). Ensemble representations have also been hypothesized to aid in texture segmentation and detection of a deviant in a scene (Cavanagh, 2001).

Few explanations for crowding have proposed that it might also produce a beneficial by-product. One model proposed by Parkes and colleagues in 2001, however, suggested that crowding arises because the visual system automatically pools, or averages, visual features over space. When observers made ensemble orientation judgments, the orientation of a crowded Gabor patch was nevertheless precisely pooled into the perceived ensemble orientation (Parkes et al., 2001). If crowding and ensemble perception share a common mechanism, changes in the degree of crowding should result in a concordant shift in ensemble perception.

In the present experiments, we examined whether the degree of ensemble pooling, defined as an observer's perception of the average orientation information in the stimulus array, is independent of the degree of crowding. Experiment 1 measured crowding in the UVF and LVF. The strength of crowding was manipulated while holding constant other factors known to strongly modulate this effect (e.g., density and eccentricity). In Experiment 2, we directly measured ensemble perception, using the same approach and stimuli. This allowed us to test whether the degree of integration between the individual elements - the ensemble percept (Fig. 1) - would scale with the crowding effect. Although it is possible to get ensemble perception

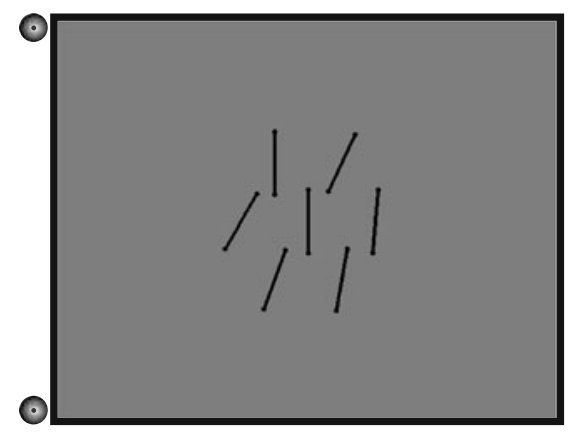

Fig. 1 Demonstration of crowding and ensemble perception. When viewed in peripheral vision, the orientation of the central target bar is difficult to identify, due to crowding by closely surrounding flankers. However, an observer is easily able to discriminate the average tilt of all the bars-ensemble perception. We measured crowding and ensemble perception in the upper and lower visual fields, using a stimulus similar to that shown in the figure to test the hypothesis that crowding facilitates ensemble perception without crowding, this study addressed the question of whether crowding adds an extra benefit. If crowding facilitates ensemble perception, estimates of ensemble orientation should become more accurate in the UVF as crowding becomes stronger.

\section{Experiment 1}

Crowding is asymmetric in the UVF and LVF (He et al., 1996). In Experiment 1, we investigated whether a crowding paradigm might also reveal visual-field-dependent differences in the degree to which flanker orientation biases target judgments. If common mechanisms drive both processes, the biasing effect of flanker orientation should also vary across the UVF and LVF.

\section{Method}

Four observers ( 1 female, 3 male) from the University of California, Davis participated. All the participants were experienced psychophysical observers, and 3 were naïve as to the purpose of the experiment. All had normal or corrected-to-normal vision. The data were collected in the same sessions as data for another experiment on visually guided reaching.

Stimuli were presented on a Toshiba Regza LCD monitor with a display resolution of $1,024 \times 768$ pixels and a refresh rate of $60 \mathrm{~Hz}$. An iMac computer running MATLAB (The Math Works Inc., Natick, MA) and Psychophysics Toolbox (Brainard, 1997; Pelli, 1997) controlled stimulus presentation. The observers used a chinrest while viewing a monitor placed $51.5 \mathrm{~cm}$ away. A $1 / 4$-in. sheet of Plexiglas covered the screen.

The stimulus consisted of a black central target bar surrounded by an equidistant radial array of six flanker bars of identical size and color, placed at $0^{\circ}, 60^{\circ}, 120^{\circ}, 180^{\circ}$, $240^{\circ}$, and $300^{\circ}$ around the target (see Fig. 1). Each bar was $4.4^{\circ}$ long and $0.24^{\circ}$ wide, with a rounded top $0.35^{\circ}$ in diameter. The background luminance of the monitor was $125 \mathrm{~cd} / \mathrm{m}^{2}$, and the luminance of the bars was $0.22 \mathrm{~cd} / \mathrm{m}^{2}$. Observers wore an eye patch to ensure monocular fixation (right eye) on a small LED mounted to the left side of the monitor throughout the trial block.

The stimulus always appeared to the right of fixation. The center of the target was separated $18.1^{\circ}$ horizontally and $19.7^{\circ}$ vertically from the fixation point, and the target could be in the UVF or LVF (equidistant to fixation point). The stimulus appeared in the same location, relative to the screen, for both LVF and UVF discriminations (the fixation point was moved between two locations). The density of the array (center-to-center spacing of the flankers and 
central target) was varied from $4.3^{\circ}$ (most crowded) to $10.7^{\circ}$ (least crowded) in four steps of $1.6^{\circ}$. The orientation of the central target bar was set at $5^{\circ}, 0^{\circ}$, or $-5^{\circ}$ from vertical, randomly on each trial. Flanking bars were manipulated independently of the target, with each having a random orientation within $30^{\circ}$ of vertical in intervals of $5^{\circ}$. The mean, or ensemble, orientation of the six flanker bars on each trial ranged from $-15^{\circ}$ to $15^{\circ}$ about vertical in intervals of $5^{\circ}$. The orientation of the flankers was randomly generated on each trial and gave no information about the orientation of the target. In each trial block, observers were presented with an equal number of all the possible target and mean flanker orientation combinations. The order of testing in the UVF and LVF was randomized for each observer. Participants made 630 total judgments $(2$ visual fields $\times 5$ flanker densities $\times 7$ ensemble orientations $\times$ 3 target orientations $\times 3$ trials).

Observers triggered each trial by pressing the space bar on a keyboard. The stimulus appeared for $500 \mathrm{~ms}$ or until the participant removed his or her hand from the space bar to make a response. Observers made a threealternative forced choice (3AFC) keypress corresponding to the three possible target orientations (leftward tilt, rightward tilt, or vertical).

On separate control trials, a target appeared in isolation. Otherwise, the control trials were identical in timing and procedure to the experimental trials. Each of the four control trial blocks in the UVF and LVF consisted of 15 trials (5 trials at each of the three target orientations).

\section{Results}

Figure 2 plots the group and individual observer data for crowded orientation discriminations in the LVF (solid line) and UVF (dotted line). A 2 (visual field) $\times 5$ (density) $\times 7$ (ensemble orientation) $\times 3$ (target orientation) ANOVA

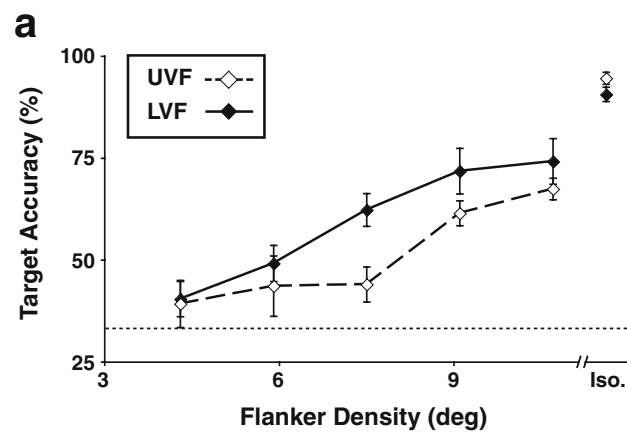

Fig 2 Crowding in the upper visual field (UVF) and the lower visual field (LVF) for the group (a) and individual observers (b). Plotted is orientation discrimination performance as a function of flanker density in the LVF (solid line) and UVF (dashed line). "Iso" indicates performance in the isolated target control condition. Individual confirmed that crowding was greater in the UVF, $F(1,3)=$ 15.7, $p<.05, \eta^{2}=.84$. There were also significant main effects of density, such that less dense arrays were less crowded, $F(4,12)=13.7, p<.01, \eta^{2}=.82$, and of mean ensemble orientation, where target accuracy was highest with maximum ensemble tilt, $F(6,18)=2.7, p=.05, \eta^{2}=$ .73. Lastly, there was a significant interaction between visual field and density, reflecting the rightward shift in the UVF discriminations apparent in Fig. 2a, $F(4,12)=6.4, p<.01$, $\eta^{2}=.68$. To estimate the $66.7 \%$ correct threshold for the group as a whole, we used MATLAB's "pfit" function, running 10,000 bootstrap simulations (Monte Carlo) on the 4 observers' combined data. To improve the fit of this simulation, data from all the participants were treated as if they had come from a single observer. In the LVF, a flanker density of $8.8^{\circ}$ was needed to reach the $67 \%$ threshold, while in the UVF, the same threshold was $10.4^{\circ}$. This difference was statistically significant, $p<.01$.

Control trials, where observers judged the orientation of a bar in isolation, showed that differences in crowding reported above were not due to differences in acuity or task difficulty. Discrimination performance for an isolated target was similar in the UVF and LVF : $90.7 \%$ in the LVF and $94.6 \%$ in the UVF. This difference was not significant, $t(3)=2.6, p=.08$.

How the orientation of flanker bars biased the perception of the crowded target provides insight into the relationship between crowding and integration of flanker information. Figure 3 plots the correlation between target judgments and the mean orientation of the six flanker bars, for each of the five densities tested in the LVF and UVF. Target judgments were biased by the ensemble flanker orientation when the density of the stimuli increased (smaller target-flanker spacing), $F=12.6, p<.01, \eta^{2}=.808$. However, while crowding was more pronounced in the UVF, a 2 (visual field $) \times 5$ (density) ANOVA revealed no difference between the degree that ensemble information was utilized across

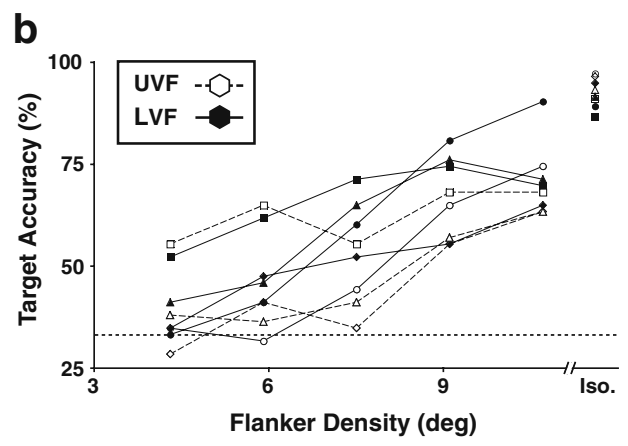

observer data are plotted with closed symbols for discriminations in the LVF and with open symbols for the UVF. The dashed horizontal line represents chance performance in the three-alternative forced choice task. Error bars indicate standard errors 


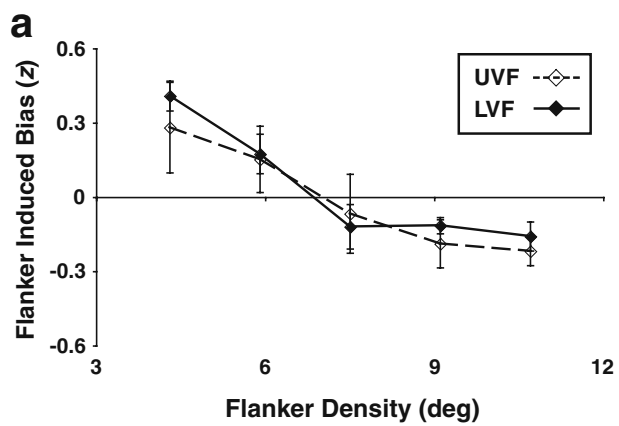

Fig. 3 Measure of flanker-orientation-induced bias during crowded target judgments in the upper visual field (UVF) and the lower visual field (LVF) for the group (a) and individual participants (b). Plotted are Fisher $z$ correlations between observers' target discriminations and the average orientation of the flanker array for the LVF (closed

visual fields, $F(1,3)=0.24$, n.s. When the orientation of the target bar was included in this ensemble average (six flanker bars plus target), there continued to be no difference in observers' use of the ensemble orientation in the UVF and LVF, $F(1,3)=0.37$, n.s. Across visual fields and densities, when the target's orientation is added to the ensemble, it increases the bias presented in Fig. 3 in the expected positive direction by $0.03 z$ units, $F(1,3)=70.2$, $p<.01, \eta^{2}=.959$, confirming that both target and average flanker orientation contributed to perceived target orientation.

\section{Experiment 2}

Although Experiment 1 suggested a dissociation between crowding and the bias introduced by the average flanker orientation, it did not directly tap into ensemble perception, because observers were instructed only to report on, and focus their attention toward, the central target. The goal of Experiment 2 was to directly measure ensemble perception in the UVF and LVF using the same stimuli as in Experiment 1.

\section{Method}

Four observers (1 female, 3 male) participated in this experiment. Two participants in Experiment 1 also participated in this experiment. Three were naïve as to the purpose of the experiment.

The method in Experiment 2 was identical to that in Experiment 1, with the following two exceptions. First, observers were instructed to report the average orientation of the entire ensemble of bars (six radial bars and one central bar). Second, we used a $2 \mathrm{AFC}$ design, instead of a $3 \mathrm{AFC}$ design; observers discriminated whether the entire ensemble was tilted left or right of vertical. To equate the

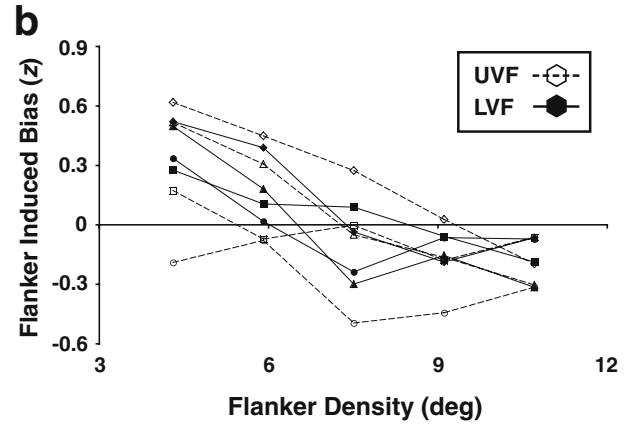

symbols, solid line) and UVF (dashed line, open symbols). Positive values signify that observers' responses were biased toward the average flanker orientation. There was no significant difference between the degree of ensemble averaging between the LVF and UVF. Error bars indicate standard errors

testing conditions across Experiments 1 and 2, trials on which the ensemble orientation summed to vertical $\left(0^{\circ}\right)$ were presented but were excluded from analysis because of the low number of those trials ( 15 per visual field). This left 600 total judgments for Experiment 2 (20 trials $\times 3$ trial blocks $\times 5$ flanker densities $\times 2$ visual fields).

\section{Results}

Figure 4 presents the participants' accuracy in discriminating the ensemble orientation in both the UVF and LVF, averaged across the five stimulus densities tested. As was expected, accuracy of ensemble orientation judgments improved with increasing degree of ensemble tilt, $F(9,27)=56.1, p<.01, \eta^{2}=.949$. Importantly, a 2 (visual field) $\times 5$ (density) ANOVA revealed that visual field had no effect on the accuracy of participants' judgments of the average ensemble orientation, $F(1,3)=$ 0.31 , n.s. Furthermore, there was no significant interaction between visual field and density, $F(4,12)=1.2$, n.s. To

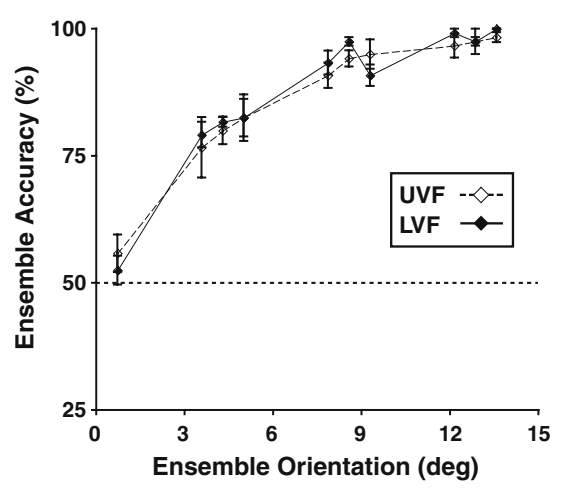

Fig. 4 Ensemble orientation task. Plotted is the group accuracy summed across the five ensemble densities tested. The dashed horizontal line represents chance performance in the two-alternative forced choice task. Error bars indicate standard errors 
compare this ensemble effect with that of crowding in Experiment 1, we separately analyzed the middle ensemble density of $5.9^{\circ}$ - the density that had shown the largest effect between visual fields on crowding judgments in Experiment 1. A 2 (visual field) $\times 10$ (ensemble orientation) ANOVA showed no effect of visual field on ensemble perception at a density of $5.9^{\circ}, F(1,3)=0.06$, n.s. Lastly, the manipulation of display density in this ensemble task had little effect on ensemble judgments, $F(4,12)=1.6$, n.s.

\section{Discussion}

This study compared crowded orientation discriminations with ensemble orientation judgments in the UVF and LVF. Experiment 1 showed higher spatial resolution (less crowding) in the lower visual field, replicating $\mathrm{He}$ et al. (1996). Interestingly, there was a strong influence of flanker orientation on participants' reports of target orientation; however, this bias did not differ across the UVF and LVF, unlike crowding. Using the same stimuli, Experiment 2 specifically tested whether an observer's ensemble percept-a measure of the ability to integrate orientation information across space-also shared crowding's asymmetry across the UVF and LVF. The degree of flanker integration did not differ across the UVF and LVF. Furthermore, stimulus density differentially affected the flanker-induced bias in Experiment 1 and ensemble perception in Experiment 2. This dissociation, potentially owing to the different task strategies employed across tasks, may be taken as further evidence that judgments of crowded orientation and ensemble orientation are not the same. Although crowding and ensemble perception are dissociable processes that do not fully covary in the context of these findings, this does not imply that they are always completely separable processes (i.e., some aspects of crowding may, in fact, overlap with those of ensemble perception).

Although our second experiment confirmed that participants perceive the average orientation in a crowd of tilted features (Parkes et al., 2001), the biasing effect of the flankers on the perceived target orientation reported in Experiment 1 (Fig. 3) reveals both attractive and repulsive effects; targets were reported as being more similar to the flankers in dense displays but different from or in contrast to the flankers at larger separations. This was despite the target being crowded to some degree at all the densities tested (i.e., the accuracy at the largest target-flanker separation was lower than isolated target performance as seen in Fig. 2). Intriguingly, this is in some ways phenomenologically similar to tilt capture and contrast illusions, which may have center-surround organization (Clifford, 2002; Clifford, Wenderoth, \& Spehar, 2000;
Schwartz, Hsu, \& Dayan, 2007). Similar findings with a rod-and-frame stimulus have also demonstrated that the orientation of a central target is either captured or repulsed by the frame, depending on its orientation (Beh, Wenderoth, \& Purcell, 1971). Attempts to reconcile both crowding and the tilt illusion under a single opponency model have had some success (e.g., Solomon, Felisberti \& Morgan, 2004). Our experiments suggest that future work may need to consider crowding, tilt contrast, and pooling as interacting effects.

The idea that "crowding and texture perception are opposite sides of the same coin," is an interesting hypothesis, put forth by Parkes et al. (2001), because it suggests that crowding may be, in part, beneficial. They found that observers account for the orientation of a crowded target when discriminating the tilt of an array of Gabors and suggested "that 'crowding' is simply the name we give to texture perception when we do not wish it to occur." This ability to extract an ensemble or average from a set of objects provides a benefit to the observer, since crowding may help by making ensembles easier to compute. Our results - that variation in crowding is not always accompanied by variation in the ensemble percept - suggest that this relationship does not always hold. Our findings suggest that crowding can degrade resolution, while not conferring much benefit to the observer.

The relationship between crowding and ensemble perception has been probed in previous research. However, the question of whether crowding adds some extra benefit has been unanswered. To answer that, we looked for covariation in crowding and ensemble perception. Livne and Sagi (2007) showed that the crowding effect is modulated by changing the configuration of flankers (smooth, interrupted, or "sun" patterned), while holding the overall orientation of the array constant. This finding is in line with that in Banks and Prinzmetal (1976), who, although not specifically testing crowding, demonstrated that changes in the flanker configuration affected reaction times in a peripheral search and detection task. Furthermore, Nandy and Tjan (2007) found that errors in recognizing a crowded target were not always predicted by ensemble pooling alone. Recently, Dakin, Bex, Cass, and Watt (2009) showed that the addition of crowding elements in an ensemble orientation task disrupts local orientation estimates, but not the absolute number of elements that can be integrated. Collectively, these results hint that the processes underlying flanker interference in crowding are more sophisticated than compulsory pooling of the flanker features. Unlike the present study, however, these studies did not test the specific hypothesis that crowding can facilitate ensemble perception.

The finding of Livne and Sagi (2007) that there is less crowding with some configurations suggests that ensembles 
can be computed before crowding, or that crowding can operate at different levels. In fact, both crowding and ensemble perception can occur selectively at different levels of visual analysis. Crowding operates not just on low-level features, but also on high-level object representations such as upright faces (Farzin et al., 2009; Louie et al., 2007). Crowding therefore reflects the limits of visual processing, due to both integrative and competitive interference from flanker items at multiple levels of the visual hierarchy. Ensemble perception, in turn, also occurs at multiple levels-including low-level features (Alvarez \& Oliva, 2008; Ariely, 2001, Chong \& Treisman, 2003; Parkes et al., 2001; Watamaniuk \& Sekuler, 1992) and representations of high-level objects such as faces (Haberman \& Whitney, 2007, 2009).

Crowding has a deleterious effect on many perceptual tasks: limiting letter recognition (Bouma, 1970; Yu, Cheung, Legge, \& Chung, 2007) and reading speed (Chung, 2002; Pelli et al., 2007), slowing eye movements during search (Vlaskamp \& Hooge, 2006), impairing the precision of grasp orientation (Bulakowski, Post, \& Whitney, 2009), and driving recognition deficits in some observers with disorders of vision, including strabismic amblyopes (Klein \& Levi, 1985). While it would be ideal if crowding conferred some benefit to perception in light of its cost, the experiments here suggest that crowding does not benefit our perception of ensembles. This does not, however, preclude crowding from serving some useful purpose in vision. Whether crowding results from low-level integration mechanisms, spatial imprecision, or the coarse resolution of higher level attentional processes, evidence supports the basic conclusion that crowding sets limits on spatial resolution in clutter. The present study suggests that a potential silver lining of crowding - that it facilitates ensemble perception - is also lost in the crowd.

\section{Author Note Supported by NIH and NSF 0748689.}

Open Access This article is distributed under the terms of the Creative Commons Attribution Noncommercial License which permits any noncommercial use, distribution, and reproduction in any medium, provided the original author(s) and source are credited.

\section{References}

Alvarez, G. A., \& Oliva, A. (2008). The representation of simple ensemble visual features outside the focus of attention. Psychological Science, 19, 392-398.

Ariely, D. (2001). Seeing sets: Representation by statistical properties. Psychological Science, 12, 157-162.

Banks, W. P., \& Prinzmetal, W. (1976). Configurational effects in visual information processing. Perception \& Psychophysics, 19, 361-367.

Beh, H. C., Wenderoth, P. M., \& Purcell, A. T. (1971). The angular function of a rod-and-frame illusion. Perception \& Psychophysics, 9, 353-355.
Bouma, H. (1970). Interaction effects in parafoveal letter recognition. Nature, 226, 177-178.

Brainard, D. H. (1997). The psychophysics toolbox. Spatial Vision, 10, 433-436.

Bulakowski, P. F., Bressler, D. W., \& Whitney, D. (2007). Shared attentional resources for global and local motion processing. Journal of Vision, 7(10, Art. 10), 1-10.

Bulakowski, P. F., Post, R. B., \& Whitney, D. (2009). Visuomotor crowding: The resolution of grasping in cluttered scenes. Frontiers in Behavioral Neuroscience, 3, 49. doi:10.3389/neuro.08.049.2009

Cavanagh, P. (2001). Seeing the forest but not the trees. Nature Neuroscience, 4, 673-674.

Chong, S. C., \& Treisman, A. (2003). Representation of statistical properties. Vision Research, 43, 393-404.

Chung, S. T. L. (2002). The effect of letter spacing on reading speed in central and peripheral vision. Investigative Ophthalmology \& Visual Science, 43, 1270.

Clifford, C. W. (2002). Perceptual adaptation: Motion parallels orientation. Trends in Cognitive Sciences, 6, 136-143.

Clifford, C. W., Wenderoth, P., \& Spehar, B. (2000). A functional angle on some after-effects in cortical vision. Proceedings of the Royal Society of LondonB, 267, 1705-1710.

Dakin, S. C., \& Watt, R. J. (1997). The computation of orientation statistics from visual texture. Vision Research, 37, 3181-3192.

Dakin, S. C., Bex, P. J., Cass, J. R., \& Watt, R. J. (2009). Dissociable effects of attention and crowding on orientation averaging. Journal of Vision, 9(11, Art. 28), 1-16.

Farzin, F., Rivera, S., \& Whitney, D. (2009). Holistic crowding of Mooney faces. Journal of Vision, 9(6, Art. 18), 1-15.

Flom, M. C., Weymouth, F. W., \& Kahneman, D. (1963). Visual resolution and contour interaction. Journal of the Optical Society of America, 53, 1026-1032.

Haberman, J., Harp, T., \& Whitney, D. (2009). Averaging facial expression over time. Journal of Vision, 9(11, Art. 1), 1-13.

Haberman, J., \& Whitney, D. (2007). Rapid extraction of mean emotion and gender from sets of faces. Current Biology, 17, R751-R753.

Haberman, J., \& Whitney, D. (2009). Seeing the mean: Ensemble coding for sets of faces. Journal of Experimental Psychology: Human Perception and Performance, 35, 718-734.

He, S., Cavanagh, P., \& Intriligator, J. (1996). Attentional resolution and the locus of visual awareness. Nature, 383, 334-337.

Klein, S. A., \& Levi, D. M. (1985). Vernier acuity, crowding and amblyopia. Vision Research, 25, 979-991.

Korte, W. (1923). Uber die Gestaltauffassung im indirekten Sehen. Zeitschrift für Psychologie, 93, 17-82.

Levi, D. M. (2008). Crowding-An essential bottleneck for object recognition: A mini-review. Vision Research, 48, 635-654.

Livne, T., \& Sagi, D. (2007). Configuration influence on crowding. Journal of Vision, 7(2, Art. 4), 1-12.

Louie, E. G., Bressler, D. W., \& Whitney, D. (2007). Holistic crowding: Selective interference between configural representations of faces in crowded scenes. Journal of Vision, 7(2, Art. 24), 1-11.

Nandy, A. S., \& Tjan, B. S. (2007). The nature of letter crowding as revealed by first- and second-order classification images. Journal of Vision, 7(2, Art. 5), 1-26.

Parkes, L., Lund, J., Angelucci, A., Solomon, J. A., \& Morgan, M. (2001). Compulsory averaging of crowded orientation signals in human vision. Nature Neuroscience, 4, 739-744.

Pelli, D. G. (1997). The VideoToolbox software for visual psychophysics: Transforming numbers into movies. Spatial Vision, 10, 437-442.

Pelli, D. G. (2008). Crowding: A cortical constraint on object recognition. Current Opinion in Neurobiology, 18, 445-451.

Pelli, D. G., Palomares, M., \& Majaj, N. J. (2004). Crowding is unlike ordinary masking: Distinguishing feature integration from detection. Journal of Vision, 4(12, Art. 12), 1136-1169. 
Pelli, D. G., Tillman, K. A., Freeman, J., Su, M., Berger, T. D., \& Majaj, N. J. (2007). Crowding and eccentricity determine reading rate. Journal of Vision, 7(2, Art. 20), 1-36.

Schwartz, O., Hsu, A., \& Dayan, P. (2007). Space and time in visual context. Nature Reviews. Neuroscience, 8, 522-535.

Solomon, J. A., Felisberti, F. M., \& Morgan, M. J. (2004). Crowding and the tilt illusion: Toward a unified account. Journal of Vision, 4(6, Art. 9), 500-508.

Strasburger, H. (2005). Unfocussed spatial attention underlies the crowding effect in indirect form vision. Journal of Vision, 5(11, Art. 8), 1024-1037.

Stuart, J. A., \& Burian, H. M. (1962). A study of separation difficulty: Its relationship to visual acuity in normal and amblyopic eyes. American Journal of Ophthalmology, 53, 471-477.
Townsend, J. T., Taylor, S. G., \& Brown, D. R. (1971). Lateral masking for letters with unlimited viewing time. Perception \& Psychophysics, 10, 375-378.

Vlaskamp, B. N., \& Hooge, I. T. (2006). Crowding degrades saccadic search performance. Vision Research, 46, 417-425.

Watamaniuk, S. N. J., \& Sekuler, R. (1992). Temporal and spatial integration in dynamic random-dot stimuli. Vision Research, 32, 2341-2347.

Williams, D. W., \& Sekuler, R. (1984). Coherent global motion percepts from stochastic local motions. Vision Research, 24, 5562.

Yu, D., Cheung, S., Legge, G., \& Chung, S. T. L. (2007). Effect of letter spacing on visual span and reading speed. Journal of Vision, 7(2, Art. 2), 1-10. 